\title{
The EffectLiteR Approach for Analyzing Average and Conditional Effects - Supplementary Materials
}

\author{
Axel Mayer ${ }^{\mathrm{a}}$, Lisa Dietzfelbinger ${ }^{\mathrm{b}}$, Yves Rosseel $^{\mathrm{a}}$, Rolf Steyer ${ }^{\mathrm{b}}$ \\ ${ }^{a}$ Department of Data Analysis, Faculty of Psychology and Educational Sciences, Ghent University \\ ${ }^{b}$ Department of Methodology and Evaluation Research, Institute of Psychology, University of Jena
}

\section{Supplement A: The EffectLiteR Software Package}

\section{A.1 Basic Commands}

EffectliteR is an R package ( $\mathrm{R}$ Core Team, 2013) that can be used to estimate average and conditional effects of a treatment variable on an outcome variable, taking into account multiple continuous and categorical covariates. EffectLiteR also includes a graphical user interface based on the shiny package (RStudio and Inc., 2014). The R package is available from CRAN and can be installed as usual provided that a recent version of $\mathrm{R}$ is installed:

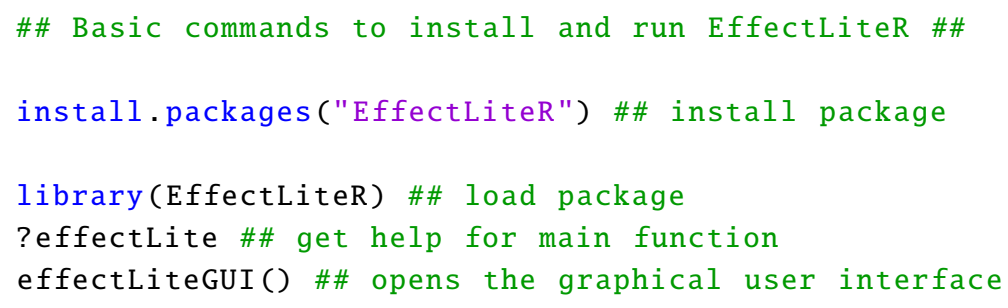

Users who wish to try out EffectLiteR without installing any software can use the online version of the graphical user interface at https://amayer.shinyapps.io/elrshiny/. The development version of the package along with descriptions of how to install this version is available from https://github.com/amayer2010. 


\section{A.2 Screenshot of the Graphical User Interface}

\section{EffectLiteR}

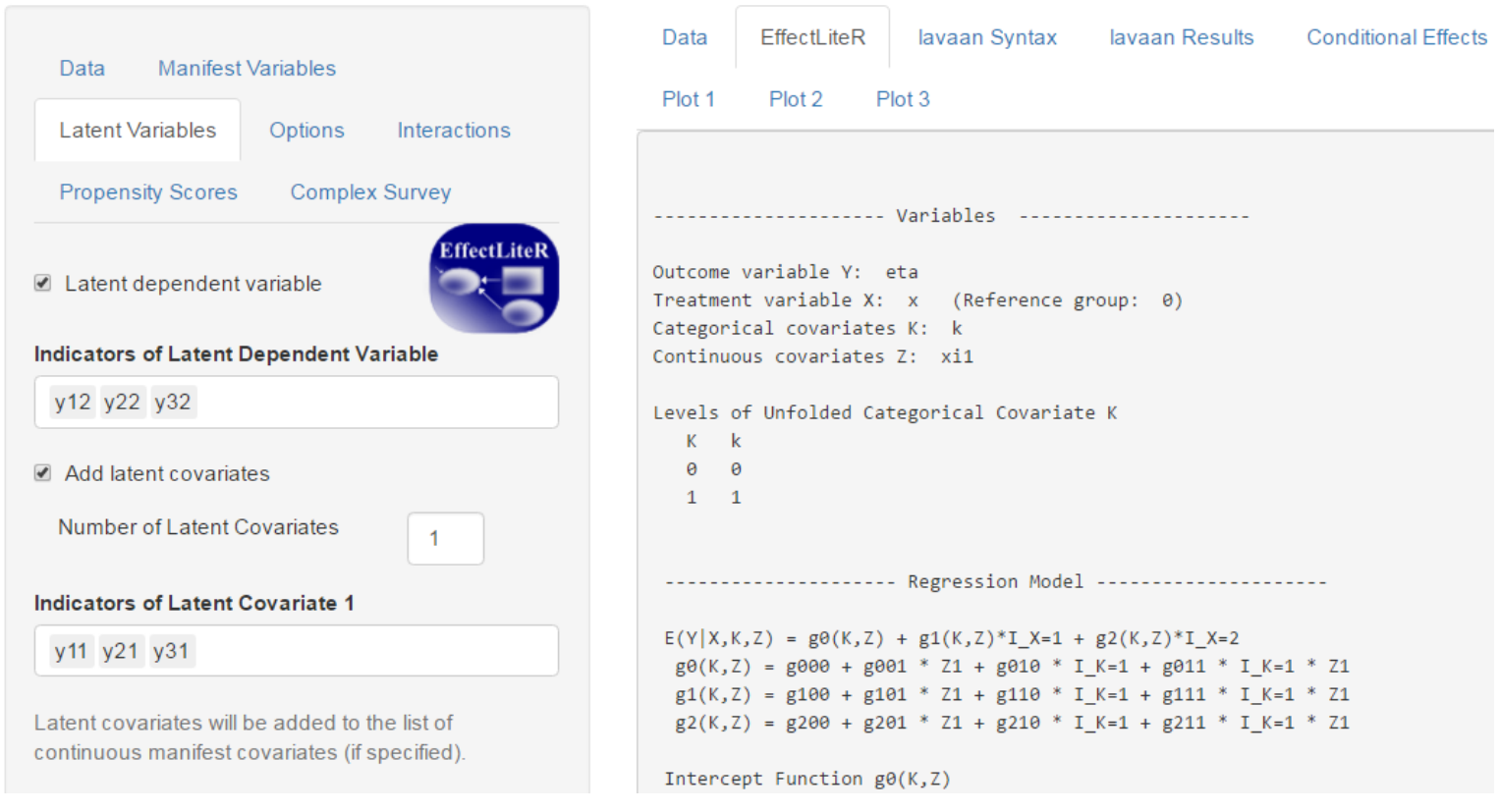

\section{A.3 EffectliteR Input Syntax}

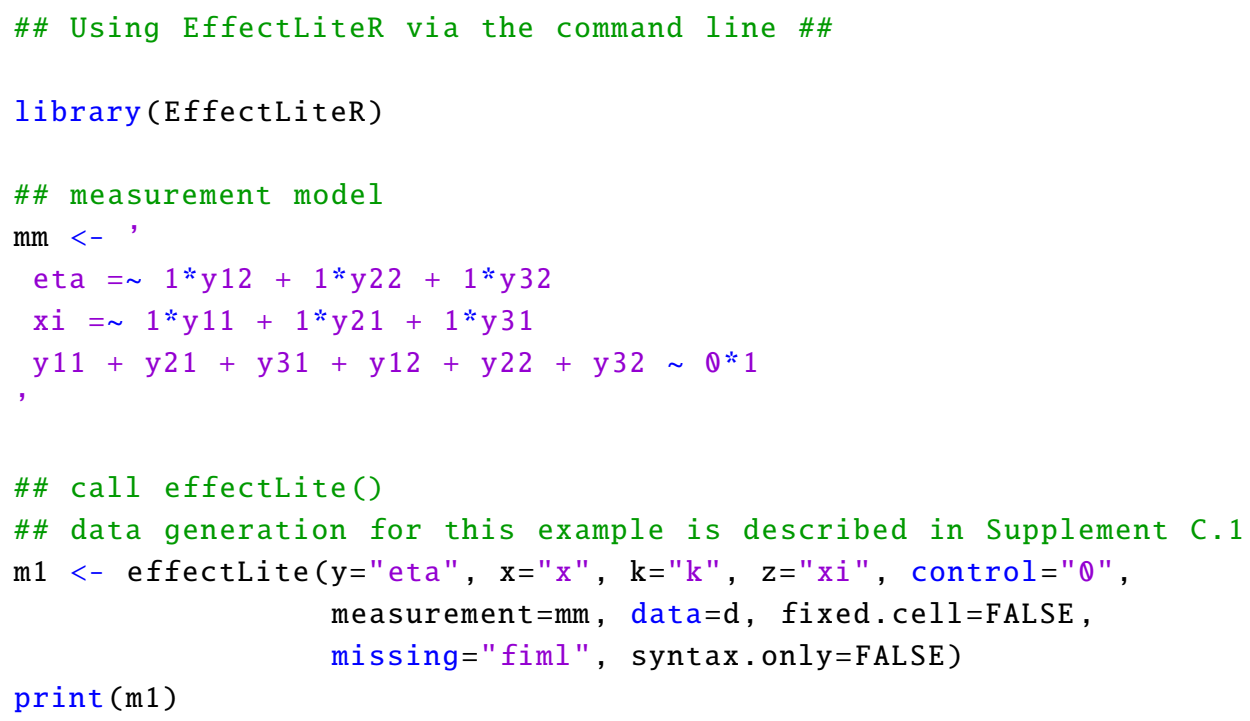




\section{A.4 Excerpts from EffectliteR Output}

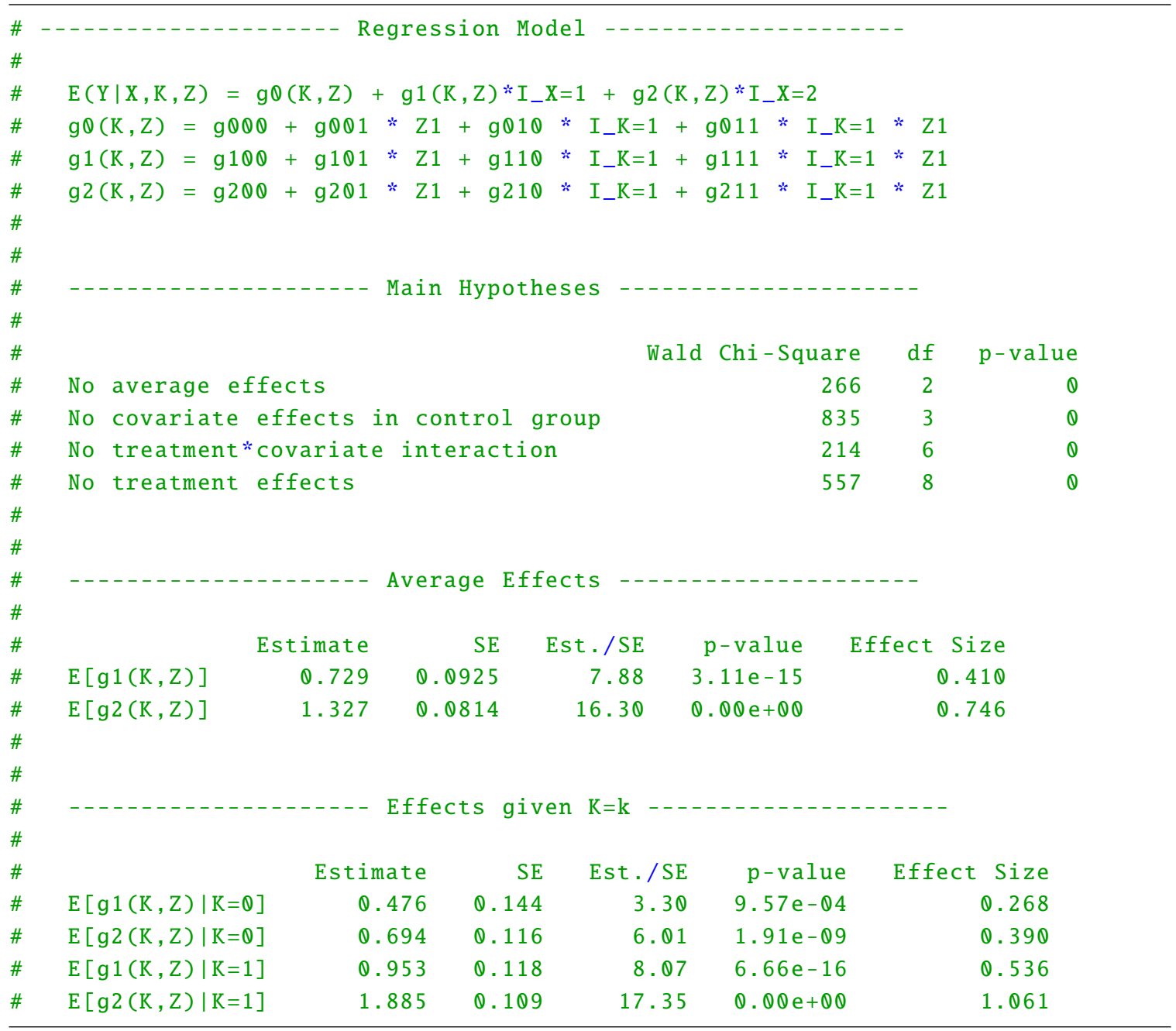




\section{Supplement B: Complete Computations for the Motivating Example}

\section{B.1 Multigroup SEM with Stochastic Group Sizes for the Simulated Data Example}

Group-invariant $\tau$-equivalent measurement model

$$
\left(\begin{array}{l}
Y_{12} \\
Y_{22} \\
Y_{32} \\
Y_{11} \\
Y_{21} \\
Y_{31}
\end{array}\right)=\left(\begin{array}{l}
0 \\
0 \\
0 \\
0 \\
0 \\
0
\end{array}\right)+\left(\begin{array}{ll}
1 & 0 \\
1 & 0 \\
1 & 0 \\
0 & 1 \\
0 & 1 \\
0 & 1
\end{array}\right)\left(\begin{array}{l}
\eta \\
\xi
\end{array}\right)+\left(\begin{array}{l}
\varepsilon_{12} \\
\varepsilon_{22} \\
\varepsilon_{32} \\
\varepsilon_{11} \\
\varepsilon_{21} \\
\varepsilon_{31}
\end{array}\right)
$$

Group-specific structural model in each of the six groups $(3 \times 2)$

$$
\left(\begin{array}{l}
\eta \\
\xi
\end{array}\right)=\left(\begin{array}{c}
\alpha_{x k 0} \\
\mu_{x k 1}
\end{array}\right)+\left(\begin{array}{cc}
0 & \alpha_{x k 1} \\
0 & 0
\end{array}\right)\left(\begin{array}{l}
\eta \\
\xi
\end{array}\right)+\left(\begin{array}{l}
\zeta_{0} \\
\zeta_{1}
\end{array}\right)
$$

Group sizes for each of the six groups $(3 \times 2)$

$$
\log \left(n_{x k}\right)=\kappa_{x k}
$$

\section{B.2 Effect Functions}

Based on the parameters $\alpha_{x k z}, \mu_{x k 1}$, and $\kappa_{x k}$ of the multigroup structural equation model, we can identify average and conditional effects. The parameterization of the regression $E(\eta \mid X, K, \xi)$ is given by:

$$
\begin{gathered}
E(\eta \mid X, K, \xi)=g_{0}(K, \xi)+g_{1}(K, \xi) \cdot I_{X=1}+g_{2}(K, \xi) \cdot I_{X=2} \\
g_{0}(K, \xi)=\gamma_{000}+\gamma_{010} \cdot K+\gamma_{001} \cdot \xi+\gamma_{011} \cdot K \cdot \xi \\
g_{1}(K, \xi)=\gamma_{100}+\gamma_{110} \cdot K+\gamma_{101} \cdot \xi+\gamma_{111} \cdot K \cdot \xi \\
g_{2}(K, \xi)=\gamma_{200}+\gamma_{210} \cdot K+\gamma_{201} \cdot \xi+\gamma_{211} \cdot K \cdot \xi
\end{gathered}
$$

First, we compute the regression coefficients of the effect funtions $g_{1}(K, \xi)$ and $g_{2}(K, \xi)$ following Equations 17 and 18:

$$
\begin{array}{ll}
\gamma_{100}=\alpha_{100}-\alpha_{000} & \gamma_{200}=\alpha_{200}-\alpha_{000} \\
\gamma_{101}=\alpha_{101}-\alpha_{001} & \gamma_{201}=\alpha_{201}-\alpha_{001} \\
\gamma_{110}=\left(\alpha_{110}-\alpha_{100}\right)-\left(\alpha_{010}-\alpha_{000}\right) & \gamma_{210}=\left(\alpha_{210}-\alpha_{200}\right)-\left(\alpha_{010}-\alpha_{000}\right) \\
\gamma_{111}=\left(\alpha_{111}-\alpha_{101}\right)-\left(\alpha_{011}-\alpha_{001}\right) & \gamma_{211}=\left(\alpha_{211}-\alpha_{201}\right)-\left(\alpha_{011}-\alpha_{001}\right)
\end{array}
$$




\section{B.3 Average and Conditional Effects}

Average Effects $A E_{t 0}$ : In our example, there are two average effects, $A E_{10}$, the average effect of treatment $X=1$ vs. $X=0$, and $A E_{20}$, the average effect of treatment $X=2$ vs. $X=0$. Following Table 3, these are identified as:

$$
\begin{aligned}
& E\left[g_{1}(K, \xi)\right]=\gamma_{100}+\gamma_{110} \cdot E(K)+\gamma_{101} \cdot E(\xi)+\gamma_{111} \cdot E(K \cdot \xi) \\
& E\left[g_{2}(K, \xi)\right]=\gamma_{200}+\gamma_{210} \cdot E(K)+\gamma_{201} \cdot E(\xi)+\gamma_{211} \cdot E(K \cdot \xi)
\end{aligned}
$$

In order to compute the average effects based on model parameters, we need to compute the unconditional expectations of covariates and product terms (cf. Table 2):

$$
\begin{aligned}
P(X=x, K=k)= & \exp \left(\kappa_{x k}\right) /\left[\exp \left(\kappa_{00}\right)+\exp \left(\kappa_{01}\right)+\exp \left(\kappa_{10}\right)+\exp \left(\kappa_{11}\right)+\exp \left(\kappa_{20}\right)+\exp \left(\kappa_{21}\right)\right] \\
E(\xi)= & \mu_{001} \cdot P(X=0, K=0)+\mu_{011} \cdot P(X=0, K=1)+\mu_{101} \cdot P(X=1, K=0)+ \\
& +\mu_{111} \cdot P(X=1, K=1)+\mu_{201} \cdot P(X=2, K=0)+\mu_{211} \cdot P(X=2, K=1) \\
P(K=0)= & P(X=0, K=0)+P(X=1, K=0)+P(X=2, K=0) \\
P(K=1)= & P(X=0, K=1)+P(X=1, K=1)+P(X=2, K=1) \\
E\left(\xi I_{K=1}\right)= & \mu_{011} \cdot P(X=0, K=1)+\mu_{111} \cdot P(X=1, K=1)+\mu_{211} \cdot P(X=2, K=1)
\end{aligned}
$$

Then, the average effects are identified as functions of the regression coefficients of the effect functions and the unconditional expectations of covariates and product terms.

Conditional Effects $C E_{t 0 ; K=k}$ : In our example, there are four $(K=k)$-conditional effects, the two conditional effects of treatment $X=1$ vs. $X=0$ for males $C E_{10 ; K=0}$ and females $C E_{10 ; K=1}$, and the two conditional effects of treatment $X=2$ vs. $X=0$ for males $C E_{20 ; K=0}$ and females $C E_{20 ; K=1}$. Following Table 3, these are identified as:

$$
\begin{aligned}
& E\left[g_{1}(K, \xi) \mid K=0\right]=\gamma_{100}+\gamma_{101} \cdot E(\xi \mid K=0) \\
& E\left[g_{1}(K, \xi) \mid K=1\right]=\gamma_{100}+\gamma_{110}+\gamma_{101} \cdot E(\xi \mid K=1)+\gamma_{111} \cdot E(\xi \mid K=1) \\
& E\left[g_{2}(K, \xi) \mid K=0\right]=\gamma_{200}+\gamma_{201} \cdot E(\xi \mid K=0) \\
& E\left[g_{2}(K, \xi) \mid K=1\right]=\gamma_{200}+\gamma_{210}+\gamma_{201} \cdot E(\xi \mid K=1)+\gamma_{211} \cdot E(\xi \mid K=1)
\end{aligned}
$$

For the identification of the four $(K=k)$-conditional effects, we need the regression coefficients of the effect functions (see above) and the ( $K=k$ )-conditional expectations of $\xi$ :

$$
\begin{aligned}
P(X=x \mid K=k) & =P(X=x, K=k) / P(K=k) \\
E(\xi \mid K=0) & =\mu_{001} \cdot P(X=0 \mid K=0)+\mu_{101} \cdot P(X=1 \mid K=0)+\mu_{201} \cdot P(X=2 \mid K=0) \\
E(\xi \mid K=1) & =\mu_{011} \cdot P(X=0 \mid K=1)+\mu_{111} \cdot P(X=1 \mid K=1)+\mu_{211} \cdot P(X=2 \mid K=1),
\end{aligned}
$$

where $P(K=k)$ and $P(X=x, K=k)$ have already been computed in the section about the identification of average effects. 
Conditional Effects $C E_{t 0 ; X=x}$ : There are six different kinds of $(X=x)$-conditional effects in our example, i.e., the three conditional effects of treatment $X=1$ vs. $X=0$ given values $x$ of $X$, and the three conditional effects of treatment $X=2$ vs. $X=0$ given values $x$ of $X$ :

$$
\begin{aligned}
& E\left[g_{1}(K, \xi) \mid X=0\right]=\gamma_{100}+\gamma_{110} \cdot E\left(I_{K=1} \mid X=0\right)+\gamma_{101} \cdot E(\xi \mid X=0)+\gamma_{111} \cdot E\left(\xi I_{K=1} \mid X=0\right) \\
& E\left[g_{1}(K, \xi) \mid X=1\right]=\gamma_{100}+\gamma_{110} \cdot E\left(I_{K=1} \mid X=1\right)+\gamma_{101} \cdot E(\xi \mid X=1)+\gamma_{111} \cdot E\left(\xi I_{K=1} \mid X=1\right) \\
& E\left[g_{1}(K, \xi) \mid X=2\right]=\gamma_{100}+\gamma_{110} \cdot E\left(I_{K=1} \mid X=2\right)+\gamma_{101} \cdot E(\xi \mid X=2)+\gamma_{111} \cdot E\left(\xi I_{K=1} \mid X=2\right) \\
& E\left[g_{2}(K, \xi) \mid X=0\right]=\gamma_{200}+\gamma_{210} \cdot E\left(I_{K=1} \mid X=0\right)+\gamma_{201} \cdot E(\xi \mid X=0)+\gamma_{211} \cdot E\left(\xi I_{K=1} \mid X=0\right) \\
& E\left[g_{2}(K, \xi) \mid X=1\right]=\gamma_{200}+\gamma_{210} \cdot E\left(I_{K=1} \mid X=1\right)+\gamma_{201} \cdot E(\xi \mid X=1)+\gamma_{211} \cdot E\left(\xi I_{K=1} \mid X=1\right) \\
& E\left[g_{2}(K, \xi) \mid X=2\right]=\gamma_{200}+\gamma_{210} \cdot E\left(I_{K=1} \mid X=2\right)+\gamma_{201} \cdot E(\xi \mid X=2)+\gamma_{211} \cdot E\left(\xi I_{K=1} \mid X=2\right)
\end{aligned}
$$

In order to compute the $(X=x)$-conditional effects, we also need the $(X=x)$-conditional expectations of covariates and product terms:

$$
\begin{aligned}
E\left(I_{K=k} \mid X=x\right) & =P(K=k \mid X=x)=P(X=x, K=k) / P(X=x) \\
E(\xi \mid X=0) & =\mu_{001} \cdot P(K=0 \mid X=0)+\mu_{011} \cdot P(K=1 \mid X=0) \\
E(\xi \mid X=1) & =\mu_{101} \cdot P(K=0 \mid X=1)+\mu_{111} \cdot P(K=1 \mid X=1) \\
E(\xi \mid X=2) & =\mu_{201} \cdot P(K=0 \mid X=2)+\mu_{211} \cdot P(K=1 \mid X=2) \\
E\left(\xi I_{K=1} \mid X=0\right) & =\mu_{011} \cdot P(K=1 \mid X=0) \\
E\left(\xi I_{K=1} \mid X=1\right) & =\mu_{111} \cdot P(K=1 \mid X=1) \\
E\left(\xi I_{K=1} \mid X=2\right) & =\mu_{211} \cdot P(K=1 \mid X=2)
\end{aligned}
$$

Conditional Effects $C E_{t 0 ; X=x, K=k}$ : Finally, we consider $(X=x, K=k)$-conditional effects. In our simulated example, there are twelve different such effects, i.e.,

$$
\begin{array}{llll}
E\left[g_{1}(K, \xi) \mid X=0, K=0\right], & E\left[g_{1}(K, \xi) \mid X=0, K=1\right], & E\left[g_{1}(K, \xi) \mid X=1, K=0\right], \\
E\left[g_{1}(K, \xi) \mid X=1, K=1\right], & E\left[g_{1}(K, \xi) \mid X=2, K=0\right], & E\left[g_{1}(K, \xi) \mid X=2, K=1\right], \\
E\left[g_{2}(K, \xi) \mid X=0, K=0\right], & E\left[g_{2}(K, \xi) \mid X=0, K=1\right], & E\left[g_{2}(K, \xi) \mid X=1, K=0\right], \\
E\left[g_{2}(K, \xi) \mid X=1, K=1\right], & E\left[g_{2}(K, \xi) \mid X=2, K=0\right], & E\left[g_{2}(K, \xi) \mid X=2, K=1\right] .
\end{array}
$$

These effects can directly be computed based on regression coefficients of the effect functions and the model parameters for $(X=x, K=k)$-conditional expectations of the continuous latent covariate:

$$
\begin{aligned}
& E\left[g_{1}(K, \xi) \mid X=x, K=0\right]=\gamma_{100}+\gamma_{101} \cdot \mu_{x 01} \\
& E\left[g_{1}(K, \xi) \mid X=x, K=1\right]=\gamma_{100}+\gamma_{110}+\gamma_{101} \cdot \mu_{x 11}+\gamma_{111} \cdot \mu_{x 11} \\
& E\left[g_{2}(K, \xi) \mid X=x, K=0\right]=\gamma_{200}+\gamma_{201} \cdot \mu_{x 01} \\
& E\left[g_{2}(K, \xi) \mid X=x, K=1\right]=\gamma_{200}+\gamma_{210}+\gamma_{201} \cdot \mu_{x 11}+\gamma_{211} \cdot \mu_{x 11}
\end{aligned}
$$




\section{Supplement C: Data Generation}

\section{C.1 R Syntax to Generate Data}

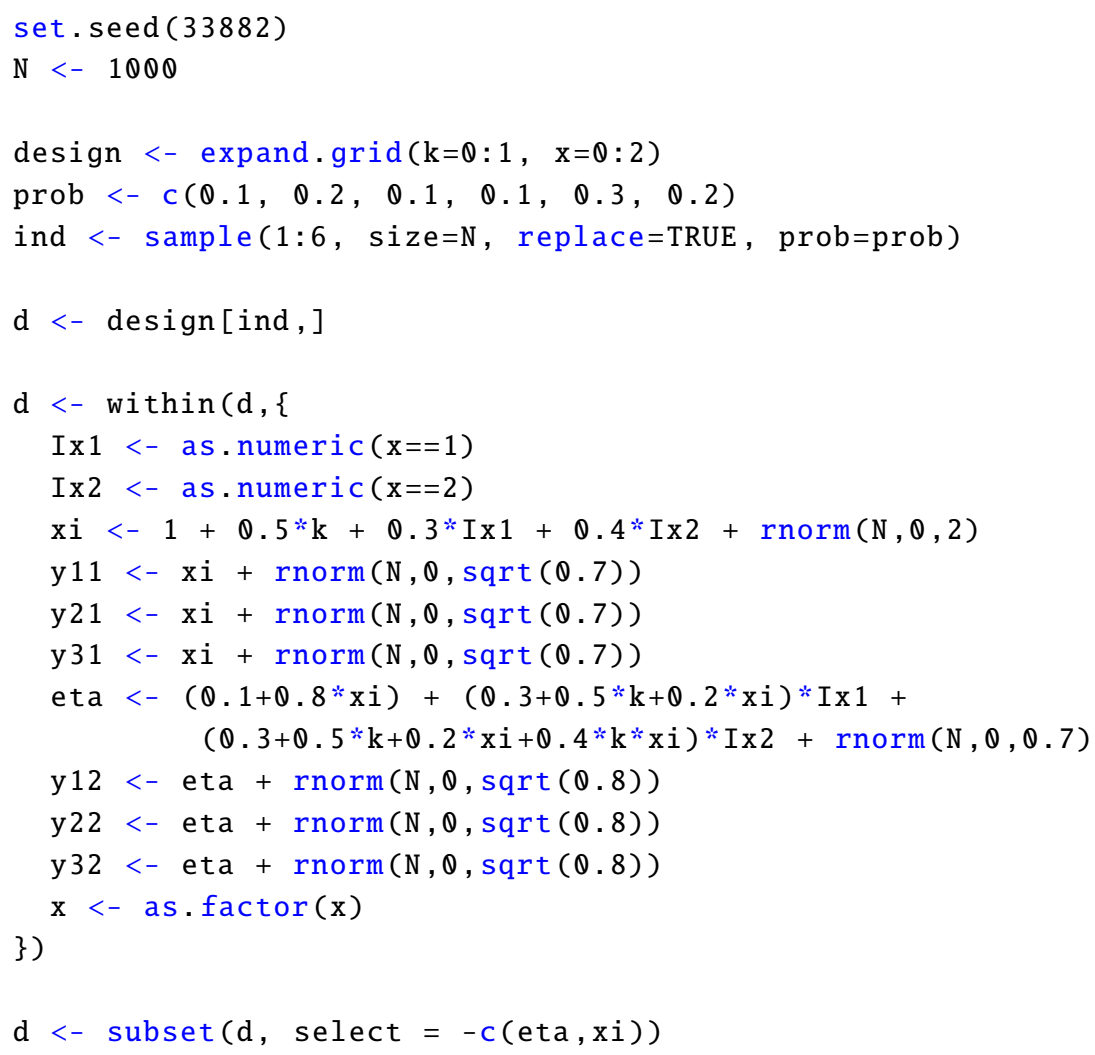




\section{C.2 True Population Parameters for Data Generation}

Cell and marginal probabilities:

\begin{tabular}{lccc} 
& $K=0$ & $K=1$ & All \\
\hline$X=0$ & 0.1 & 0.2 & 0.3 \\
$X=1$ & 0.1 & 0.1 & 0.2 \\
$X=2$ & 0.3 & 0.2 & 0.5 \\
\hline All & 0.5 & 0.5 & 1.0
\end{tabular}

Manifest variables:

$$
\begin{array}{ll}
Y_{11}=\xi+\varepsilon_{11} & Y_{12}=\eta+\varepsilon_{12} \\
Y_{21}=\xi+\varepsilon_{21} & Y_{22}=\eta+\varepsilon_{22} \\
Y_{31}=\xi+\varepsilon_{31} & Y_{32}=\eta+\varepsilon_{32}
\end{array}
$$

where $\operatorname{Var}\left(\varepsilon_{11}\right)=\operatorname{Var}\left(\varepsilon_{21}\right)=\operatorname{Var}\left(\varepsilon_{31}\right)=0.7$ and $\operatorname{Var}\left(\varepsilon_{12}\right)=\operatorname{Var}\left(\varepsilon_{22}\right)=\operatorname{Var}\left(\varepsilon_{32}\right)=0.8$.

\section{Latent variables:}

$$
\begin{aligned}
\xi= & 1+0.5 \cdot K+0.3 \cdot I_{X=1}+0.4 \cdot I_{X=2}+\zeta_{1} \\
\eta= & (0.1+0.8 \cdot \xi)+(0.3+0.5 \cdot K+0.2 \cdot \xi) \cdot I_{X=1} \\
& +(0.3+0.5 \cdot K+0.2 \cdot \xi+0.4 \cdot K \cdot \xi) \cdot I_{X=2}+\zeta_{0}
\end{aligned}
$$

where $\operatorname{Var}\left(\zeta_{1}\right)=4$ and $\operatorname{Var}\left(\zeta_{0}\right)=0.49$. 


\section{C.3 True Average and Conditional Effects}

True average and conditional effects of conventional therapy ( $X=1$ vs. $X=0$ ) and of innovative therapy ( $X=2$ vs. $X=0$ ) compared to the control group in the simulated data example.

\begin{tabular}{|c|c|c|c|}
\hline Effect & True value & Effect & True value \\
\hline \multicolumn{4}{|c|}{ True Average and Conditional Effects of Conventional Therapy } \\
\hline \multicolumn{2}{|c|}{ Average Effect $A E_{10}$} & \multicolumn{2}{|c|}{ Conditional Effects $C E_{10 ; X=x}$} \\
\hline$A E_{10}$ & 0.852 & $C E_{10 ; X=0}$ & 0.9 \\
\hline \multicolumn{2}{|c|}{ Effect Function $g_{1}(K, \xi)$} & $C E_{10 ; X=1}$ & 0.86 \\
\hline$\gamma_{100}$ & 0.3 & $C E_{10 ; X=2}$ & 0.82 \\
\hline$\gamma_{101}$ & 0.2 & \multicolumn{2}{|c|}{ Conditional Effects $C E_{10 ; X=x, K=k}$} \\
\hline$\gamma_{110}$ & 0.5 & $C E_{10 ; X=0, K=0}$ & 0.5 \\
\hline$\gamma_{111}$ & 0 & $C E_{10 ; X=1, K=0}$ & 0.56 \\
\hline \multicolumn{2}{|c|}{ Conditional Effects $C E_{10 ; K=k}$} & $C E_{10 ; X=2, K=0}$ & 0.58 \\
\hline$C E_{10 ; K=0}$ & 0.56 & $C E_{10 ; X=0, K=1}$ & 1.1 \\
\hline \multirow{2}{*}{$C E_{10 ; K=1}$} & 1.144 & $C E_{10 ; X=1, K=1}$ & 1.16 \\
\hline & & $C E_{10 ; X=2, K=1}$ & 1.18 \\
\hline \multicolumn{4}{|c|}{ True Average and Conditional Effects of Innovative Therapy } \\
\hline \multicolumn{2}{|c|}{ Average Effect $A E_{20}$} & \multicolumn{2}{|c|}{ Conditional Effects $C E_{20 ; X=x}$} \\
\hline$A E_{20}$ & 1.196 & $C E_{20 ; X=0}$ & 1.3 \\
\hline \multicolumn{2}{|c|}{ Effect Function $g_{2}(K, \xi)$} & $C E_{20 ; X=1}$ & 1.22 \\
\hline$\gamma_{200}$ & 0.3 & $C E_{20 ; X=2}$ & 1.24 \\
\hline$\gamma_{201}$ & 0.2 & \multicolumn{2}{|c|}{ Conditional Effects $C E_{20 ; X=x, K=k}$} \\
\hline$\gamma_{210}$ & 0.5 & $C E_{20 ; X=0, K=0}$ & 0.5 \\
\hline$\gamma_{211}$ & 0.4 & $C E_{20 ; X=1, K=0}$ & 0.56 \\
\hline \multicolumn{2}{|c|}{ Conditional Effects $C E_{20 ; K=k}$} & $C E_{20 ; X=2, K=0}$ & 0.58 \\
\hline$C E_{20 ; K=0}$ & 0.56 & $C E_{20 ; X=0, K=1}$ & 1.7 \\
\hline \multirow[t]{2}{*}{$C E_{20 ; K=1}$} & 1.832 & $C E_{20 ; X=1, K=1}$ & 1.88 \\
\hline & & $C E_{20 ; X=2, K=1}$ & 1.94 \\
\hline
\end{tabular}

The true effect functions are given by $g_{t}(K, \xi)=\gamma_{t 00}+\gamma_{t 10} \cdot K+\gamma_{t 01} \cdot \xi+\gamma_{t 11} \cdot K \cdot \xi$ for $t=1,2$. The reported effects are true average effects $A E_{t 0}=E\left[g_{t}(K, \xi)\right]$, conditional effects given gender $C E_{t 0 ; K=k}=E\left[g_{t}(K, \xi) \mid K=k\right]$, conditional effects given a treatment condition $C E_{t 0 ; X=x}=E\left[g_{t}(K, \xi) \mid X=x\right]$ and conditional effects given gender and a treatment condition $C E_{t 0 ; X=x, K=k}=E\left[g_{t}(K, \xi) \mid X=x, K=k\right]$. 


\section{Supplement D: Monte Carlo Study for Sample Size Considerations}

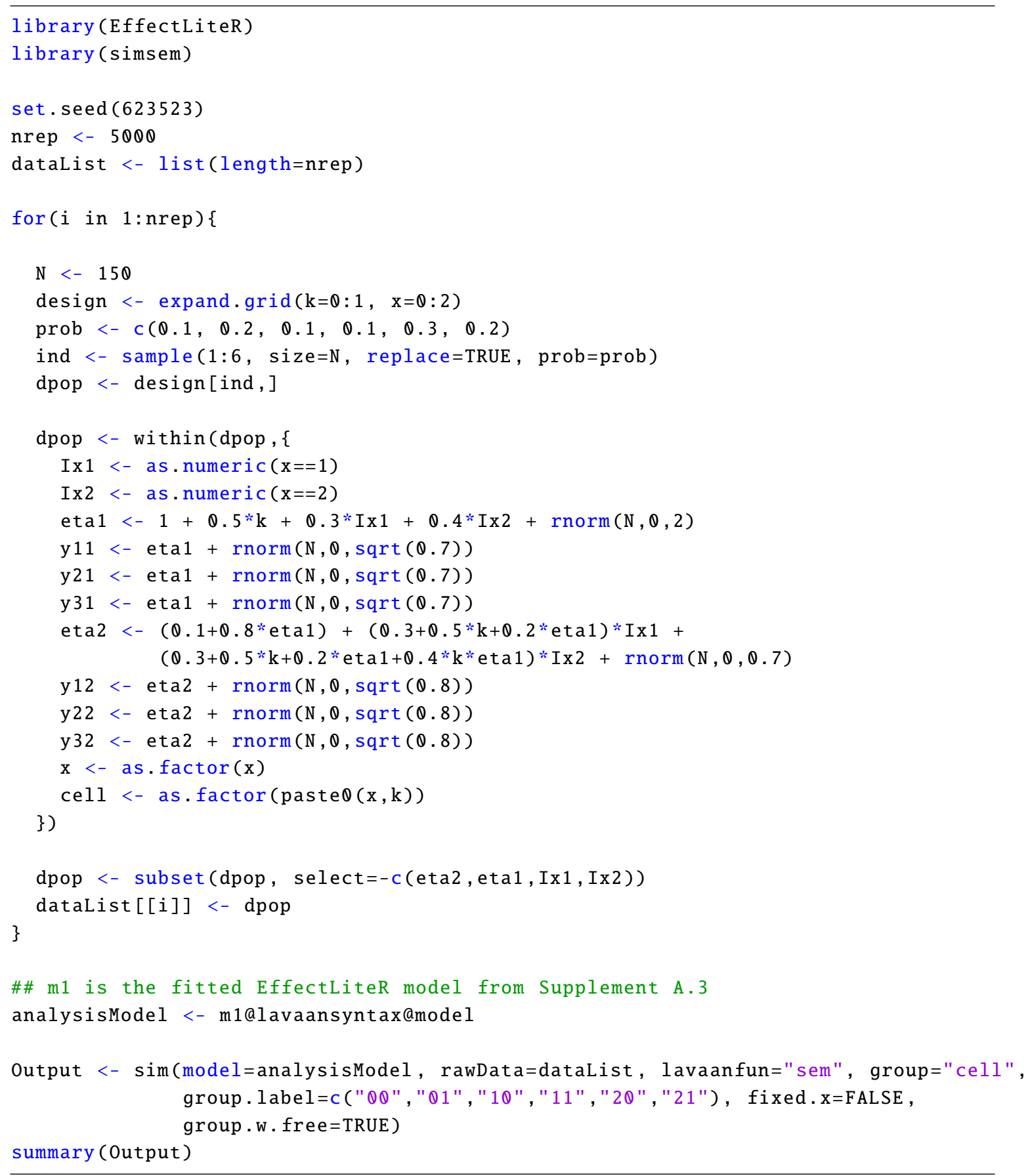




\section{References}

R Core Team. (2013). R: A language and environment for statistical computing [Computer software manual]. Vienna, Austria. Retrieved from http://www.R-project.org/

RStudio and Inc. (2014). shiny: Web application framework for R [Computer software manual]. Retrieved from http: //CRAN. R-project. org/package=shiny (R package version 0.10 .1$)$ 